\title{
Leather Inspection based on Wavelets
}

\author{
João Luís Sobral \\ Departamento de Informática, Universidade do Minho \\ 4710 - 057 Braga, Portugal \\ jls@di.uminho.pt
}

\begin{abstract}
This paper presents a new methodology to detect leather defects, based on the wavelet transform. The methodology uses a bank of optimised filters, where each filter is tuned to one defect type. Filter shape and wavelet sub-band are selected based the maximisation of the ratio between features values on defect regions and on normal regions. The proposed methodology can detect defects even when small features variations are present, which are not detect by generic texture classification techniques, and is fast enough to be used for real-time leather inspection.
\end{abstract}

\section{Introduction}

Leather inspection has been recognised as one very complex problem on the area of texture classification. Like most of natural textures, feature values have a high variation, forming a pseudo-random structure, e.g., features present a high variation but still follow a statistical distribution. Machine made textures have a much more regular pattern, since features values follow a more predefined range.

Generic texture classification methods assign a texture to one of a set of predefined classes. Typical texture features for each texture class are computed from texture samples. Comparing the texture features with the features from each texture class, using some proximity criterion, can classify an unknown texture.

Defect detection is usually based on a simpler texture classification scheme, using just a defect class and a normal class. The classification can be based on a threshold classifier that assigns a texture to one of these two classes. Unfortunately, the difference among features in defect types can be large, leading to several defect classes and to a more complex classifier. Also, features difference from normal and defective regions can be very small, leading to a poor performance when using generic texture classification schemes.

This paper presents a methodology to select a set of optimised filters, where each filter is applied to the texture wavelet transform. The process searches for filters that achieve the highest ratio between features on normal and defective regions for each defect type. Each filter is based on a wavelet sub-band and can have different size and orientation.

The rest of this paper is organised as follows. Section 2 presents an overview of related work, mainly based on filter bank and wavelet based approaches. Section 3 introduces the methodology and section 4 presents performance results. Section 5 closes the paper with suggestions for future work. 


\section{Related work}

The filter bank approach is one of the most used approaches for texture classification. It is based on a bank of convolution masks to extract texture features. Gabor filters [1] have been used for texture classification because of their optimal space/frequency. Wavelet transforms [2] are very efficient to calculate and have also been used for texture classification [3][4]. A comparative study of filter bank approaches for texture classification can be found in [5].

Optimised techniques for texture classification are based on filters that maximise the features difference among textures. In [6] a method is presented to design an optimal finite impulse filter for defect detection in textured materials and [7] uses a combination of Wavelets and Co-Occurrence Matrices. Optimised Gabor filters also have been used in [8][9].

\section{Proposed methodology}

This section begins by presenting wavelet-based methodologies for texture classification, then the defect detection methodology is presented and finally the process of selecting the optimised filters is analysed.

\subsection{Wavelet texture classification}

Texture classification based on wavelet transform is based on a successive application of a low pass and a high pass filter and on sub-sampling after each filter application. There are several wavelets, each one uses different low pass and high pass filters. The simplest and fastest wavelet is the Haar Wavelet, which has a low pass filter equal to $[0.5,0.5]$ and the high pass filter equal to $[-0.5,+0.5]$.

\begin{tabular}{|c|c|c|}
\hline $\begin{array}{l}\text { 1xly } \\
\text { 1xly }\end{array}$ & $\begin{array}{l}\text { lxly } \\
\text { hxly }\end{array}$ & \multirow{2}{*}{ hxly } \\
\hline $\begin{array}{l}\text { lxly } \\
\text { lxhy }\end{array}$ & $\begin{array}{c}\text { lxly } \\
\text { hxhy }\end{array}$ & \\
\hline \multicolumn{2}{|c|}{ lxhy } & hxhy \\
\hline
\end{tabular}

Fig. 1. Sub-band representation

Figure 1 shows a sub-band representation of a two level wavelet transform and the sequence of filters applied on each sub-band. For example, the top right sub-band is obtained applying a high pass filter in the horizontal direction (hx), sub-sampling to retain half of the pixels, applying a low pass filter in the vertical direction (ly) and sub-sampling again. Texture classification can be performed using the average energy on each sub-band as texture features. In this example there are 7 features per texture. 


\subsection{Defect detection}

Leather defects can have several shapes and sizes depending on the defect type. Namely, there are small defects with a high feature difference from normal areas and there are large defects that have a small feature difference from normal areas. This means that using a single filter size does not achieve a good performance: small defects require small filter sizes and large defects require larger filter sizes. Also, defect shapes are not the same for all defect types, some defects occupy a long and tiny area while others occupy a small or large square area.

Traditional filter bank approaches (Figure 2) apply a bank of filters to the input image, followed by a nonlinear function (usually squaring) and followed by a smoothing filter (averaging). The smoothing filter size is usually calculated based on the central band-pass frequency of each filter. A classifier, assigning each texture to a class, uses the resulting feature vectors from the smoothing step.

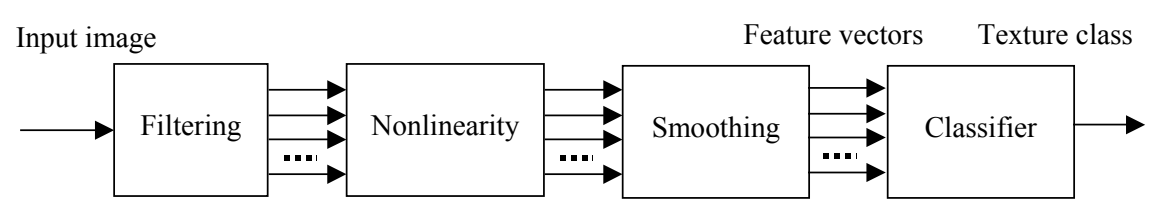

Fig. 2. Filter bank based texture classification

The proposed methodology uses a filtering step based on wavelet packets, which achieves the sub-band decomposition presented in Figure 3. This decomposition uses both the first and the second level of decomposition and generates $20(4+16)$ features per texture.
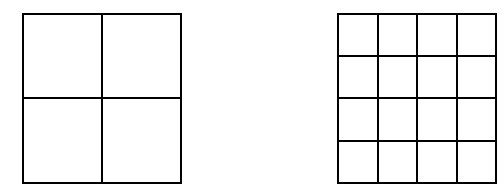

Fig. 3. Wavelet sub-band decomposition

The nonlinear processing step is not applied in this methodology, allowing the measurement of both positive and negative filter responses, improving the detection for some type of defects.

The smoothing step is based on a set of filters, each filter having a size and shape tuned to a defect type. Figure 4 presents an overview of the recognition process.

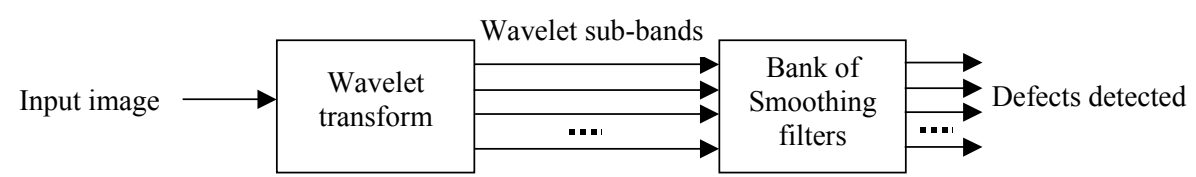

Fig. 4. Defect detection process

The wavelet transform produces 20 feature images, each filter is then applied to one of these feature images and its output will signal the detection of one defect type. Note that several filters may detect a particular defect, namely, on defects that have a high feature difference from normal regions. 


\subsection{Filter selection}

Each smoothing filter is tuned for a kind of defect. The output from an optimal filter should maximise the ratio between defect regions and normal areas. The process of selecting the best filter is based on training samples with manually identified defects.

Optimised filter selection is performed by first segmenting the image into a number of small regions. A set of predefined smoothing filters is then applied to each region and to each wavelet sub-band, obtaining a large number of features per region. The best filter is afterwards selected as the one that provides the maximum feature ratio between each defect region and the average of other regions in the same sample.

The smoothing filters perform pixel averaging over a $3 \times 3$ to $13 \times 13$ pixel neighbourhoods, both square and rectangular (e.g., 3x5, 3x7, ...). Also, for rectangular neighbourhoods, a $45^{\circ}$ rotated mask is tested. These rotated masks are more appropriated to detect thin defects that are not horizontal or vertical. Overall the process tests $2 \times 36$ filters mask, each one applied to all the wavelet sub-bands. This leads to a total of $2 \times 36 \times 20$ (e.g., 1440) possible masks, which is considerable less than other techniques [8][9], where the optimised filters have several free parameters, leading to a complex scheme to find the best filter parameters.

Smoothing filters can be viewed as a frequency subdivision of each wavelet sub-band, but since we tested several rectangular shapes and orientations the subdivision is not restricted to a square frequency notch. Also, this process tunes the filters to detect missing frequencies or positive/negative ramps (e.g. local frequencies with a high amplitude), and their size matches the expected minimum defect area. This is achieved by using minimum or maximum filter response on the defect area instead of the usual average filter response. Using minimum or maximum filter response also helps to overcome the difficulty of the accurate image segmentation.

Overall the process are based on the wavelet transform to perform a fast square partition of the frequency spectrum, followed by a finer partition, based on smoothing filters. Also note that since both the first and second levels of the wavelet transform are used, it is possible to detect defects that affect a large area of the frequency spectrum (first level), as well as defects that affect a smaller area (second level).

We found by experimental work that a ratio larger than six ensures a detect detection free from false positives (e.g., regions detected as defects that are not defects). If no filter response achieves this ratio it means that the defect cannot be detected using this kind of filters.

Each defect sample usually leads to a specific filter, so a second step selects the most generic filters. In this step the filters are successively selected based on their ability to detect the highest number of defects. This process successively selects the filter that adds the largest number of defects that are not already detected by the previously selected filters. To avoid too much filter specialisation (e.g., each filter detecting just one defect), each optimised filter is required to detect several defects.

\section{Results}

The proposed methodology has been applied to several leather samples. Each sample was taken with a resolution of five pixels per millimetre. The image brightness and 
contrast are normalised to eliminate illumination effects. It is then segmented to obtain a large amount of regions. This segmentation step selects the largest regions, which have a low brightness or a low or high contrast, which always includes defect regions and a high amount of other regions. Both filter selection (e.g., training) and leather inspection is performed using 200 regions per 4000x2000 pixels area.

We applied the proposed methodology to a database of about 150 samples. Using the undecimated Haar Wavelet transform and 8 optimised filters, it was able to achieve the same recognition rate as an experienced human operator. Filter selection was performed using another database of 20 defects. Note that many of the defects require a highly trained operator to be manually detected, since there is only a slight feature variation on the defect area, and that most of these defects are not detected by more traditional methodologies, such as Gabor filters or normal Wavelet Packets.

Figure 6 presents three small samples; the first one is detected by traditional approaches, while the second and the third are not detected. Figure 5 presents the highest ratio between the defect region and normal regions obtained on each wavelet sub-band, respectively, on the second and on the third leather sample. On both these samples the maximum ratio was obtained using a $45^{\circ}$ smoothing $7 \times 3$ filter. Note that some defects can be detected on several sub-bands. In such case, usually the lower level is preferred, since it involves a lower number of calculations. Also note that, in these two cases, using a square mask (or a non-rotated) does not achieve a ratio high enough to provide a reliable detection.

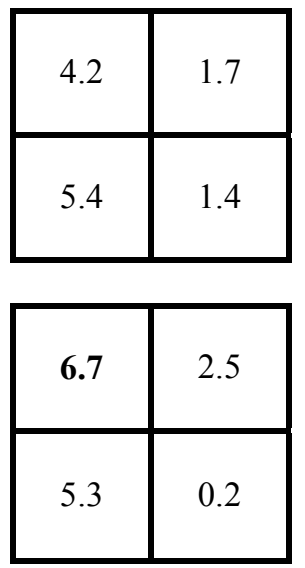

\begin{tabular}{|l|l|l|l|}
\hline 4.4 & 1.7 & 2.3 & 3.0 \\
\hline $\mathbf{6 . 1}$ & 3.0 & 2.1 & 5.3 \\
\hline $\mathbf{5 . 9}$ & 2.4 & 1.5 & 3.1 \\
\hline 4.3 & 5.1 & 3.3 & 2.7 \\
\hline $\mathbf{6 . 6}$ & 3.5 & 3.9 & 0.7 \\
\hline 5.6 & 1.7 & 1.7 & 1.0 \\
\hline 5.6 & 1.9 & 0.8 & 0.1 \\
\hline 3.3 & 0.6 & 0.6 & 0.7 \\
\hline
\end{tabular}

Fig. 5. Maximum ratio defect / normal region per wavelet sub-band

In spite of having a similar look, the third defect is larger than the second, so it requires a different mask and can be detected on the first level, in the lxly sub-band. The second defect can be detected in the lxlylxhy or in the lxhylxly sub-band.

The inspection process takes 70 seconds for an image of 4000x6000 pixels (leather size of $0.8 \mathrm{~m} \times 1.2 \mathrm{~m}$ ), which is less than the time required for an automatic machine to cut the leather. These times were collected a PC AMD XP1800+, running Red Hat Linux 8.0 and the GNU Gcc 3.2 compiler. 

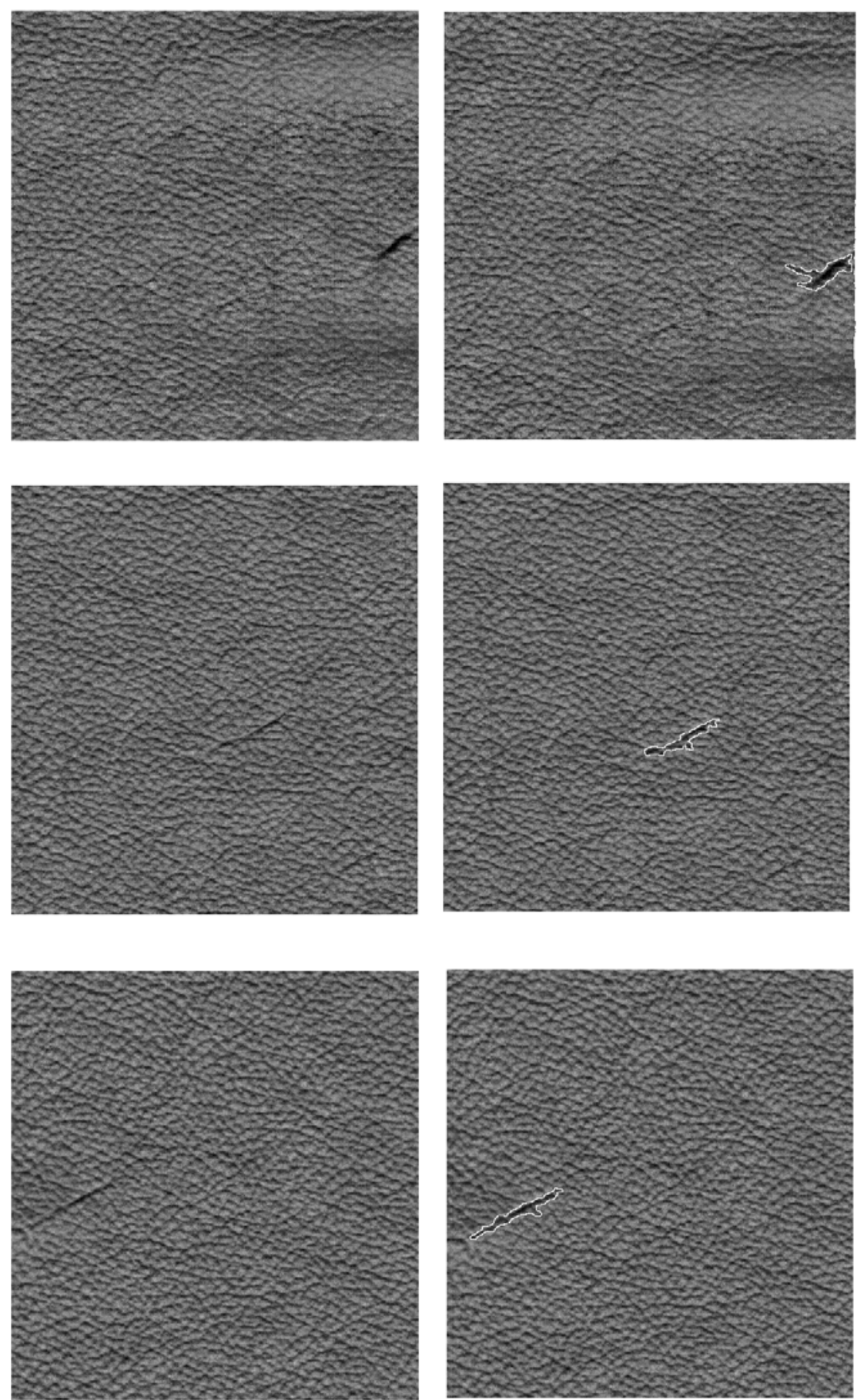

Fig. 6. Leather samples and inspection results 


\section{Conclusion}

This paper presented a methodology for leather inspection. The methodology is based on a wavelet transform and on a bank of optimised smoothing filters, each filter tuned for a particular defect type.

The methodology was able to detect several types of defects that current approaches cannot detect, it achieves the same detection rate as an experienced operator and it is fast enough to be used for real time inspection.

This methodology was applied to leather inspection but we think that it can be also applied to other inspection areas.

Current research includes using more features per optimised filter, since each filter is based on a single wavelet sub-band. This improvement should increase the recognition rate but it will also increase the complexity of the process. Ongoing work also includes experiments with other Wavelet Transforms and Gaussian smoothing filters. However all these enhancements will increase the processing time so other improvements are required to achieve the same processing time.

\section{References}

1. Jain, A., Farrokhnia, F.: Unsupervised Texture Segmentation Using Gabor Filters, IEEE International Conference on Systems, Man and Cybernetics, Nov. (1990)

2. Mallat, S.: A Theory for Multiresolution Signal Decomposition: The Wavelet Representation, IEEE Trans. Pattern Analysis and Machine Int., vol. 11(7), July (1989).

3. Laine, A., Fan, J.: Texture Classification by Wavelet Packet Signatures, IEEE Transactions on Pattern Analysis and Machine Intelligence, vol. 15(11), Nov. (1993)

4. Unser, M.: Texture Classification and Segmentation Using Wavelet Frames IEEE Transaction on Image Processing, vol. 4(11), November (1995)

5. Randen, T., Husoy, J.: Filtering for Texture Classification: A Comparative Study, IEEE Transactions on Pattern Analysis and Machine Intelligence, vol. 21(4), April (1999)

6. Kumar, A., Pang, G.: Defect Detection in Textured Materials Using Optimised Filters, IEEE Trans. Systems, Man and Cyber. - Part B: Cybernetics, vol. 32(5), October (2002)

7. Amet, A., Ertuzun, A., Ercil, A.: Texture Defect Detection using Sub-band Domain Co-Occurrence Matrices, IEEE Southwest Symposium on Image Analysis and Interpretation, April (1998)

8. Kumar, A., Pang, G.: Defect Detection in Textured Materials Using Gabor Filters, IEEE Transactions on Industry Applications, vol. 38(2), March/April (2002)

9. Bodnarova, A., Bennamoun, M., Latham, J.: A Constrained Minimisation Approach to Optimise Gabor Filters for Detecting Flaws in Woven Textiles, IEEE International Conference on Acoustics, Speech, and Signal Processing, June (2000) 